\title{
On the Survival of a Gene Represented in a Founder Population
}

\author{
C. C. Heyde
}

CSIRO Division of Mathematics and Statistics, Box 1965, P.O. Canberra City, A.C.T. 2601, Australia

\begin{abstract}
Gene survival in a population which increases without density dependence is considered using a generalization of the Moran model for haploid individuals. It is shown that situations where ultimate homozygosity is certain and where there is a non-zero probability of balanced polymorphism are both possible. Necessary and sufficient conditions in terms of the mean of the population growth distribution are given which determine which of these situations holds.
\end{abstract}

Key words: Gene survival-Founder population-Moran model-Balanced polymorphism - Ultimate homozygosity - Martingale methods

\section{Introduction and Results}

This paper deals with a founder population which is introduced into a new niche and increases without density dependence. We consider two possible alleles $a$ and $A$ at a single locus and shall address the question of the probability that the population ultimately becomes homozygous.

Suppose that we have a haploid population in which individuals die one by one at random and are replaced by new individuals. At time $n$ the population consists of $X_{n} a$-alleles and $Y_{n} A$-alleles. The next death occurs at time $n+1$ and the dying individual is replaced by a random number $U_{n}=k$, say, of new individuals which are of type $a$ or $A$ with probabilities $X_{n} /\left(X_{n}+Y_{n}\right)$ and $Y_{n} /\left(X_{n}+Y_{n}\right)$ respectively. The number of new individuals has a distribution $\left\{p_{k}, k \geqslant 1\right\}$ independent of the replacement type. The probabilities of an $a$ or $A$ individual dying are also $X_{n} /\left(X_{n}+Y_{n}\right)$ and $Y_{n} /\left(X_{n}+Y_{n}\right)$, respectively, and hence the transition probabilities for the bivariate Markov chain of the process are

$$
\begin{array}{ll}
P\left(X_{n+1}=X_{n}-1, Y_{n+1}=Y_{n}+k \mid X_{n}, Y_{n}\right)=X_{n} Y_{n}\left(X_{n}+Y_{n}\right)^{-2} p_{k}, & k \geqslant 1, \\
P\left(X_{n+1}=X_{n}+k, Y_{n+1}=Y_{n}-1 \mid X_{n}, Y_{n}\right)=X_{n} Y_{n}\left(X_{n}+Y_{n}\right)^{-2} p_{k}, & k \geqslant 1, \\
P\left(X_{n+1}=X_{n}+k-1, Y_{n+1}=Y_{n} \mid X_{n}, Y_{n}\right)=X_{n}^{2}\left(X_{n}+Y_{n}\right)^{-2} p_{k}, & k \geqslant 1, \\
P\left(X_{n+1}=X_{n}, Y_{n+1}=Y_{n}+k-1 \mid X_{n}, Y_{n}\right)=Y_{n}^{2}\left(X_{n}+Y_{n}\right)^{-2} p_{k}, & k \geqslant 1 .
\end{array}
$$

This overlapping generation model is an extension of the Moran model [e.g. Moran 
(1962), Chapter IV; Ewens (1979), Chapter 3] which corresponds to the case $p_{1}=1, p_{k}=0, k>1$.

We shall write $X_{n}+Y_{n}=N_{n}$, the number of individuals in the population at time $n$ and let $\mathscr{F}_{n}$ denote the $\sigma$-field generated by $X_{k}, Y_{k}, 0 \leqslant k \leqslant n$. The initial values $X_{0}$ and $N_{0}$ will be taken as fixed constants. Note that $N_{n}=S_{n}+N_{0}$ where $S_{n}=\left(U_{1}-1\right)+\cdots+\left(U_{n}-1\right)$, the numbers of new individuals $U_{i}$ being independent and identically distributed random variables with $P\left(U_{1}=k\right)=p_{k}, k \geqslant 1$.

The evolution of the population will be studied by considering the process $\left\{Z_{n}=X_{n} N_{n}^{-1}, n \geqslant 0\right\}$ which keeps track of the proportion of $a$-individuals in the population. We have

$$
\begin{aligned}
E\left(Z_{n+1} \mid \mathscr{F}_{n}\right)= & \sum_{k=1}^{\infty}\left[\left(X_{n}-1\right) Z_{n}\left(1-Z_{n}\right)+\left(X_{n}+k\right) Z_{n}\left(1-Z_{n}\right)\right. \\
& \left.+\left(X_{n}+k-1\right) Z_{n}^{2}+X_{n}\left(1-Z_{n}\right)^{2}\right]\left(N_{n}+k-1\right)^{-1} p_{k} \\
= & Z_{n} \sum_{k=1}^{\infty}\left[\left(2 N_{n} Z_{n}+k-1\right)\left(1-Z_{n}\right)+\left(N_{n} Z_{n}+k-1\right) Z_{n}\right. \\
& \left.+N_{n}\left(1-Z_{n}\right)^{2}\right]\left(N_{n}+k-1\right)^{-1} p_{k}=Z_{n}
\end{aligned}
$$

and hence $\left\{Z_{n}, \mathscr{F}_{n}, n \geqslant 0\right\}$ is a martingale. Since $0 \leqslant Z_{n} \leqslant 1$ we have from the martingale convergence theorem that $Z_{n} \rightarrow Z$ a.s. as $n \rightarrow \infty$ for some random variable $Z$ with $0 \leqslant Z \leqslant 1$. That is, the proportion of $a$-alleles in the population approaches some limit with probability one.

Our principal question in this paper concerns the eventual genetic composition of the population. It turns out that certain ultimate homozygosity (i.e. $P(Z=0)+P(Z=1)=P(Z(1-Z)=0)=1)$ or a prospect of balanced polymorphism (i.e. $P(Z(1-Z)>0)>0)$ are both possible and the following theorem gives a complete categorization of the circumstances under which each of these can be obtained.

Theorem. If $E U_{1}=\sum_{k=1}^{\infty} k p_{k}=\infty$ or if $p_{1}=1$ (and hence $U_{j}=1$ and $N_{j}=N_{0}$ for all $j)$ then $Z(1-Z)=0$ a.s. so that there is ultimate homozygosity with probability one. On the other hand, if $1<E U_{1}<\infty$, then $P(Z(1-Z)>0)>0$, so that there is a non-zero probability of balanced polymorphism in the population.

It is interesting to contrast these results with the corresponding ones for a generalization of the Sewall-Wright model (Heyde and Seneta, 1975). Balanced polymorphism is a possibility in both contexts but under circumstances of much less extreme population growth in the present one. Here we have given a complete categorization of circumstances under which balanced polymorphism can be obtained in contrast to partial results in Heyde and Seneta (1975). However, population growth occurs by additions of independent and identically distributed random variables in the present model in contrast to a quite general process for the other case.

The results of this paper are also relevant in the context of the founder principle of Mayr [Mayr (1942), p. 237] and to the problem of the survival of a new mutant. It should be noted that, no matter what distribution is chosen for $U_{1}$, there is always a non-zero probability of ultimate homozygosity. 


\section{Proof of Theorem}

We have for $n \geqslant 0$, and after some algebra,

$$
\begin{aligned}
E\left(Z_{n+1}\left(1-Z_{n+1}\right) \mid \mathscr{F}_{n}\right)= & \frac{X_{n} Y_{n}}{N_{n}^{2}} \sum_{k=1}^{\infty}\left[\left(X_{n}-1\right)\left(Y_{n}+k\right)+\left(X_{n}+k\right)\left(Y_{n}-1\right)\right. \\
& \left.+X_{n}\left(X_{n}+k-1\right)+Y_{n}\left(Y_{n}+k-1\right)\right] \frac{p_{k}}{\left(N_{n}+k-1\right)^{2}} \\
= & Z_{n}\left(1-Z_{n}\right) \sum_{k=1}^{\infty} \frac{N_{n}^{2}+2 k N_{n}-2 N_{n}-2 k}{\left(N_{n}+k-1\right)^{2}} p_{k} \\
= & Z_{n}\left(1-Z_{n}\right)\left\{1-\sum_{k=1}^{\infty} \frac{1+k^{2}}{\left(N_{n}+k-1\right)^{2}} p_{k}\right\} .
\end{aligned}
$$

Thus,

$$
\left\{W_{n}=\frac{Z_{n}\left(1-Z_{n}\right)}{\prod_{j=0}^{n-1}\left(1-\sum_{k=1}^{\infty} \frac{1+k^{2}}{\left(N_{j}+k-1\right)^{2}} p_{k}\right)}, \mathscr{F}_{n}, n \geqslant 1\right\}
$$

is a non-negative martingale with mean $Z_{0}\left(1-Z_{0}\right)>0$. By the martingale convergence theorem, $W_{n}$ converges a.s. to some random variable $W$ with $E W \leqslant Z_{0}\left(1-Z_{0}\right)$. Of course $Z_{n}\left(1-Z_{n}\right) \rightarrow Z(1-Z)$ a.s. since $Z_{n} \rightarrow Z$ a.s. and hence $Z(1-Z)=0$ at least on the set

$$
\left\{\prod_{j=0}^{\infty}\left(1-\sum_{k=1}^{\infty} \frac{1+k^{2}}{\left(N_{j}+k-1\right)^{2}} p_{k}\right)=0\right\},
$$

i.e. on the set

$$
\left\{\sum_{j=0}^{\infty} \sum_{k=1}^{\infty} \frac{1+k^{2}}{\left(N_{j}+k-1\right)^{2}} p_{k}=\infty\right\} .
$$

On the other hand, returning to the martingale $\left\{W_{n}, \mathscr{F}_{n}, n \geqslant 1\right\}$, we note that if

$$
V=\prod_{j=0}^{\infty}\left(1-\sum_{k=1}^{\infty} \frac{1+k^{2}}{\left(N_{j}+k-1\right)^{2}} p_{k}\right),
$$

then

$$
W=Z(1-Z) V^{-1} \leqslant \frac{1}{4} V^{-1}
$$

and if $E V^{-1}<\infty$ the dominated convergence theorem enables us to conclude that

$$
E W=E\left(Z(1-Z) V^{-1}\right)=Z_{0}\left(1-Z_{0}\right)>0
$$

which ensures that $P(Z(1-Z)>0)>0$. The proof will now be completed with the aid of three lemmas.

\section{Lemma 1.}

$$
P\left(\sum_{j=0}^{\infty} \sum_{k=1}^{\infty} \frac{1+k^{2}}{\left(N_{j}+k-1\right)^{2}} p_{k}<\infty\right)=0 \text { or } 1
$$


according as

$$
\sum_{j=0}^{\infty} \sum_{k=1}^{\infty}\left(1+k^{2}\right) p_{k} E\left(N_{j}+k-1\right)^{-2}=\infty \text { or }<\infty
$$

Proof. We use similar ideas to the proof of Lemma 1 and Theorem 1 of Schuh (1980). Write

$$
A_{j}=\sum_{k=1}^{\infty}\left(1+k^{2}\right)\left(N_{j}+k-1\right)^{-2} p_{k} .
$$

We shall first show that $\left\{\sum_{j=0}^{\infty} A_{j}<\infty\right\} \in \mathscr{G}$, the tail $\sigma$-field of $U_{1}-1, U_{2}-1, \ldots$, and hence $P\left(\sum_{j=0}^{\infty} A_{j}<\infty\right)=0$ or 1 .

Let $\mathscr{G}_{n}$ be the $\sigma$-field generated by $U_{k}-1, k \geqslant n$. Then,

$$
\begin{aligned}
{\left[U_{1}\left(N_{0}+\sum_{i=2}^{n}\left(U_{i}-1\right)+k-1\right)\right]^{-2} } & \leqslant\left[N_{0}+\sum_{i=1}^{n}\left(U_{i}-1\right)+k-1\right]^{-2} \\
& \leqslant\left[N_{0}+\sum_{i=2}^{n}\left(U_{i}-1\right)+k-1\right]^{-2}
\end{aligned}
$$

for all $n \geqslant 2$ and hence

$$
\left\{\sum_{j=0}^{\infty} A_{j}<\infty\right\}=\left\{\sum_{j=2}^{\infty} \sum_{k=1}^{\infty}\left(1+k^{2}\right)\left(N_{0}+\sum_{i=2}^{n}\left(U_{i}-1\right)+k+1\right)^{-2} p_{k}<\infty\right\} \in \mathscr{G}_{2}
$$

Applying this reasoning iteratively we obtain

$$
\left\{\sum_{j=0}^{\infty} A_{j}<\infty\right\} \in \mathscr{G}_{r} \quad \text { for all } r
$$

and hence it belongs to $\mathscr{G}=\bigcap_{r=1}^{\infty} \mathscr{G}_{r}$.

Now it follows from the Borel-Cantelli lemma that

$$
P\left(\sum_{j=0}^{\infty} A_{j}<\infty\right)=0 \quad \text { implies } \quad \sum_{j=0}^{\infty} E A_{j}=\infty
$$

and consequently it suffices to show that

$$
P\left(\sum_{j=0}^{\infty} A_{j}<\infty\right)=1 \quad \text { implies } \quad \sum_{j=0}^{\infty} E A_{j}<\infty
$$

in order to complete the proof.

To establish (1) we first choose $l>0$ so large that $P\left(\sum_{j=0}^{\infty} A_{j} \leqslant l\right)>0$ and define

$$
=\min \left[n \mid \sum_{j=0}^{n} A_{j}>l\right] \quad \text { if } \quad \sum_{j=0}^{\infty} A_{j}>l, \quad=\infty \text { otherwise }
$$

so that $P\left(n^{*}=\infty\right)>0$. We have

$$
\int_{\left\{n^{*}=n\right\}}\left(N_{n}+k-1\right)^{-2} d P \leqslant\left(N_{0}+k-1\right)^{-2} P\left(n^{*}=n\right)
$$


and since $\sum_{j=0}^{n-1} A_{j} \leqslant l$ on $\left\{n^{*}=n\right\}$,

$$
\int_{\left\{n^{*}=n\right\}}\left(\sum_{j=0}^{n-1} A_{j}\right) d P \leqslant l P\left(n^{*}=n\right) .
$$

Further, since $\left\{n^{*}=n\right\}$ belongs to the $\sigma$-field generated by $U_{i}-1, i \leqslant n$, we have for $t \geqslant n$,

$$
\begin{aligned}
\int_{\left\{n^{*}=n\right\}} & \left(N_{0}+S_{t+r}+k-1\right)^{-2} d P \\
& =\int_{\left\{n^{*}=n\right\}}\left(N_{0}+k-1+\left(U_{1}-1\right)+\cdots+\left(U_{t+r}-1\right)\right)^{-2} d P \\
& \leqslant \int_{\left\{n^{*}=n\right\}}\left(N_{0}+k-1+\left(U_{t+1}-1\right)+\cdots+\left(U_{t+r}-1\right)\right)^{-2} d P \\
& \leqslant E A_{r} P\left(n^{*}=n\right)
\end{aligned}
$$

for all $r=1,2, \ldots$.

The results (2), (3) and (4) imply that

$$
\int_{\left\{n^{*}=n\right\}}\left(\sum_{j=0}^{N} A_{j}\right) d P \leqslant\left[\sum_{k=1}^{\infty} \frac{1+k^{2}}{\left(N_{0}+k-1\right)^{2}} p_{k}+l+E \sum_{j=0}^{N} A_{j}\right] P\left(n^{*}=n\right)
$$

for all $0 \leqslant n \leqslant N$ and hence

$$
\int_{\left\{n^{*} \leqslant N\right\}}\left(\sum_{j=0}^{N} A_{j}\right) d P \leqslant\left[\sum_{k=1}^{\infty} \frac{1+k^{2}}{\left(N_{0}+k-1\right)^{2}} p_{k}+l+E \sum_{j=0}^{N} A_{j}\right] P\left(n^{*} \leqslant N\right) .
$$

However, as $\sum_{j=0}^{N} A_{j} \leqslant l$ on $\left\{n^{*}>N\right\}$, we have

$$
\begin{aligned}
\int_{\left\{n^{*}>N\right\}}\left(\sum_{j=0}^{N} A_{j}\right) d P & \leqslant l P\left(n^{*}>N\right) \\
& \leqslant\left[l+\sum_{k=1}^{\infty} \frac{1+k^{2}}{\left(N_{0}+k-1\right)^{2}} p_{k}\right] P\left(n^{*}>N\right),
\end{aligned}
$$

and from (5) and (6),

$$
E \sum_{j=0}^{N} A_{j} \leqslant l+\sum_{k=1}^{\infty} \frac{1+k^{2}}{\left(N_{0}+k-1\right)^{2}} p_{k}+\left(E \sum_{j=0}^{N} A_{j}\right) P\left(n^{*} \leqslant N\right),
$$

so that

$$
P\left(n^{*}>N\right) E \sum_{j=0}^{N} A_{j} \leqslant l+\sum_{k=1}^{\infty} \frac{1+k^{2}}{\left(N_{0}+k-1\right)^{2}} p_{k}<\infty .
$$

Finally, we let $N \rightarrow \infty$ in (7) and since $P\left(n^{*}>N\right) \rightarrow P\left(n^{*}=\infty\right)>0$ we obtain

$$
E \sum_{j=0}^{\infty} A_{j}=\lim _{N \rightarrow \infty} E \sum_{j=0}^{N} A_{j}<\infty
$$

as required. This completes the proof of the lemma. 
Lemma 2. $\sum_{j=0}^{\infty} \sum_{k=1}^{\infty}\left(1+k^{2}\right) p_{k} E\left(N_{j}+k-1\right)^{-2}$ diverges if $U_{1}=1$ a.s. or $E U_{1}=\infty$ and converges if $1<E U_{1}<\infty$.

Proof. The result for $U_{1}=1$ a.s. is obvious and we shall subsequently suppose that $P\left(U_{1}>1\right)>0$. The result for $1<E U_{1}<\infty$ follows readily via a minor modification of the proof of Lemma 3 of Athreya and Karlin (1967) but this approach does not enable us to deal with the case $E U_{1}=\infty$. We shall adopt a unified approach which enables us to treat both cases.

If $X$ is a non-negative integer valued random variable with probability generating function (p.g.f.) $f$ and $r>0$, we have for $0 \leqslant s \leqslant 1$,

$$
\begin{aligned}
E\left(s^{r+X}(r+X)^{-1}\right) & =E \int_{0}^{s} t^{r+X-1} d t \\
& =\int_{0}^{s} E t^{r+X-1} d t=\int_{0}^{s} t^{r-1} f(t) d t
\end{aligned}
$$

using Fubini's theorem. Further,

$$
\int_{0}^{1} E\left(s^{r+X-1}(r+X)^{-1}\right) d s=E(r+X)^{-2}
$$

and hence

$$
\begin{aligned}
E(r+X)^{-2} & =\int_{0}^{1} s^{-1}\left(\int_{0}^{s} t^{r-1} f(t) d t\right) d s \\
& =\int_{0}^{1}\left(\int_{t}^{1} s^{-1} d s\right) t^{r-1} f(t) d t \\
& =\int_{0}^{1}\left(\log t^{-1}\right) t^{r-1} f(t) d t
\end{aligned}
$$

Now $N_{j}=N_{0}+\left(U_{1}-1\right)+\cdots+\left(U_{j}-1\right)$, so that if $f$ is the p.g.f. of $U_{1}-1$ we have from (8) that

$$
E\left(N_{j}+k-1\right)^{-2}=\int_{0}^{1}\left(\log t^{-1}\right) t^{N_{0}+k-1} f^{j}(t) d t
$$

and

$$
\begin{aligned}
& \sum_{j=0}^{\infty} \sum_{k=1}^{\infty}\left(1+k^{2}\right) p_{k} E\left(N_{j}+k-1\right)^{-2} \\
& \quad=\int_{0}^{1}\left(\log t^{-1}\right) t^{N_{0}}\left(\sum_{j=0}^{\infty} f^{j}(t)\right)\left(\sum_{k=1}^{\infty}\left(1+k^{2}\right) p_{k} t^{k-1}\right) d t \\
& \quad=\int_{0}^{1}\left(\log t^{-1}\right) t^{N_{0}}(1-f(t))^{-1}\left(2 f(t)+t^{2} f^{\prime \prime}(t)+3 t f^{\prime}(t)\right) d t
\end{aligned}
$$

since $f(t)=\sum_{k=1}^{\infty} p_{k} t^{k-1}$. 
We observe that

$$
\lim _{t \downarrow 0}\left(\log t^{-1}\right) t^{N_{0}}(1-f(t))^{-1}\left(2 f(t)+t^{2} f^{\prime \prime}(t)+3 t f^{\prime}(t)\right)=0,
$$

and as $t \uparrow 1$,

$$
t^{N_{0}} \log t^{-1} \sim 1-t
$$

while

$$
2 f(t)+t^{2} f^{\prime \prime}(t)+3 t f^{\prime}(t) \sim C f^{\prime \prime}(t)
$$

for some constant $C\left(C=1\right.$ if $E U_{1}^{2}=\infty$ for then $f^{\prime \prime}(t) \uparrow \infty$ and $f^{\prime}(t)=o\left(f^{\prime \prime}(t)\right)$ as $t \uparrow 1$ and $C$ is given by $C f^{\prime \prime}(1)=2+f^{\prime \prime}(1)+3 f^{\prime}(1)$ if $\left.E U_{1}^{2}<\infty\right)$. Thus,

$$
\sum_{j=0}^{\infty} \sum_{k=1}^{\infty}\left(1+k^{2}\right) p_{k} E\left(N_{j}+k-1\right)^{-2}<\infty \text { or }=\infty
$$

according as

$$
\int_{\varepsilon}^{1}(1-t)(1-f(t))^{-1} f^{\prime \prime}(t) d t<\infty \text { or }=\infty
$$

for some $\varepsilon, 0<\varepsilon<1$.

Now write

$$
Q(t)=(1-f(t))(1-t)^{-1}
$$

Integration by parts in (9) gives

$$
\begin{aligned}
& \int_{\varepsilon}^{1}(1-t)(1-f(t))^{-1} f^{\prime \prime}(t) d t \\
& \quad=\int_{\varepsilon}^{1}[Q(t)]^{-1} f^{\prime \prime}(t) d t \\
& \quad=1-f^{\prime}(\varepsilon)[Q(\varepsilon)]^{-1}+\int_{\varepsilon}^{1} f^{\prime}(t) Q^{-2}(t) Q^{\prime}(t) d t
\end{aligned}
$$

with $f^{\prime}(t)[Q(t)]^{-1} \rightarrow 1$ as $t \uparrow 1$ holding since

$$
Q(t)=(1-f(t))(1-t)^{-1}=f^{\prime}(\alpha(t))
$$

for some $t<\alpha(t)<1$ by the mean value theorem and in view of the monotonicity of both $Q$ and $f^{\prime}$. Then, using $f^{\prime}(t) \sim Q(t)$ as $t \uparrow 1$ we have

$$
\int_{\varepsilon}^{1} f^{\prime}(t) Q^{-2}(t) Q^{\prime}(t) d t<\infty \text { or }=\infty
$$

according as

$$
\int_{\varepsilon}^{1} Q^{\prime}(t)[Q(t)]^{-1} d t<\infty \text { or }=\infty
$$

Finally, 


$$
\int_{\varepsilon}^{1} Q^{\prime}(t)[Q(t)]^{-1} d t=[\log Q(t)]_{\varepsilon}^{1}
$$

and the result of the lemma follows via (9) and (10) since $Q(1)=f^{\prime}(1)$ is finite if and only if $E U_{1}<\infty$.

Lemma 3. $E V^{-1}<\infty$ if $1<E U_{1}<\infty$.

Proof. We now write

$$
A\left(N_{j}\right)=\sum_{k=1}^{\infty}\left(1+k^{2}\right)\left(N_{j}+k-1\right)^{-2} p_{k}
$$

so that

$$
V=\prod_{j=0}^{\infty}\left(1-A\left(N_{j}\right)\right)
$$

and

$$
V^{-1} \leqslant \exp \left[\sum_{j=0}^{\infty} A\left(N_{j}\right)\left(1-A\left(N_{j}\right)\right)^{-1}\right]
$$

since $1-x \geqslant \exp (-x /(1-x))$ for $0<x<1$. Furthermore, $A\left(N_{j}\right) \leqslant A\left(N_{0}\right)$ and hence

$$
V^{-1} \leqslant \exp \left[\left(1-A\left(N_{0}\right)\right)^{-1} \sum_{j=0}^{\infty} A\left(N_{j}\right)\right] .
$$

Now let $n_{i}, i \geqslant 0$, denote the number of zeros in the sequence $\left\{U_{j}-1, j \geqslant 1\right\}$ between the $i$ th and $(i+1)$ st non-zero elements. Then, the $n_{i}$ are independent and identically distributed random variables with

$$
P\left(n_{0}=j\right)=p_{1}^{j}\left(1-p_{1}\right), \quad j=0,1,2, \ldots
$$

and

$$
\sum_{j=0}^{\infty} A\left(N_{j}\right) \leqslant \sum_{j=0}^{\infty}\left(n_{j}+1\right) A\left(N_{0}+j\right)
$$

so that from (11),

$$
E V^{-1} \leqslant \prod_{j=0}^{\infty} E\left[\exp \left\{\left(n_{0}+1\right)\left(1-A\left(N_{0}\right)\right)^{-1} A\left(N_{0}+j\right)\right]\right.
$$

But,

$$
\begin{aligned}
E e^{\lambda\left(n_{0}+1\right)} & =e^{\lambda} \sum_{j=0}^{\infty} e^{\lambda j} p_{1}^{j}\left(1-p_{1}\right) \\
& =\left(1-p_{1}\right) e^{\lambda}\left(1-p_{1} e^{\lambda}\right)^{-1} \\
& =e^{\lambda}\left(1-p_{1}\left(1-e^{\lambda}\right)\left(1-p_{1} e^{\lambda}\right)^{-1}\right),
\end{aligned}
$$

and hence, using (13) in (12), 


$$
E V^{-1} \leqslant \exp \left[\sum_{j=0}^{\infty} A\left(N_{0}+j\right)\right] \prod_{j=0}^{\infty}\left(1-\frac{p_{1}\left(1-\exp \left[A\left(N_{0}+j\right)\right]\right)}{1-p_{1} \exp \left[A\left(N_{0}+j\right)\right]}\right)<\infty
$$

since

$$
\begin{aligned}
\sum_{j=0}^{\infty} A\left(N_{0}+j\right) & =\sum_{j=0}^{\infty} \sum_{k=1}^{\infty}\left(1+k^{2}\right)\left(N_{0}+j+k-1\right)^{-2} p_{k} \\
& =\sum_{k=1}^{\infty}\left(\sum_{j=0}^{\infty}\left(N_{0}+j+k-1\right)^{-2}\right)\left(1+k^{2}\right) p_{k} \\
& \leqslant \sum_{k=1}^{\infty}\left(1+k^{2}\right)\left(N_{0}+k-2\right)^{-1} p_{k}<\infty
\end{aligned}
$$

for $1<E U_{1}<\infty$. This establishes Lemma 3 .

To complete the proof of the theorem it only remains to note that $Z(1-Z)=0$ a.s. if

$$
\sum_{j=0}^{\infty} \sum_{k=1}^{\infty}\left(1+k^{2}\right)\left(N_{n}+k-1\right)^{-2} p_{k}=\infty \text { a.s. }
$$

which holds if $U_{1}=1$ a.s. or $E U_{1}=\infty$ using Lemmas 1 and 2. On the other hand, $P(Z(1-Z)>0)>0$ if $E V^{-1}<\infty$ and, according to Lemma 3, this holds if $1<E U_{1}<\infty$.

Acknowledgement. Some helpful comments from P. A. P. Moran are gratefully acknowledged.

\section{References}

Athreya, K. B., Karlin, S.: Limit theorems for split times of branching processes. J. Math. Mech. 17, $257-277$ (1967)

Ewens, W. J.: Mathematical population genetics. Berlin: Springer 1979

Heyde, C. C., Seneta, E.: The genetic balance between random sampling and random population size. J. Math. Biol. 1, 317-320 (1975)

Mayr, E.: Systematics and the origin of species. New York: Columbia Univ. Press 1942; New York: Reprinted by Dover Publications 1964

Moran, P. A. P.: The statistical processes of evolutionary theory. Oxford: Clarendon Press 1962

Schuh, H.-J.: Sums of i.i.d. random variables and an application to the explanation to the explosion criterion for Markov branching processes. Department of Statistics, University of Melbourne Research Report Series, No. 2, 1980

Received June 12, 1980/Revised November 11, 1980 